\title{
A circulating cell population showing both M1 and M2 monocyte/macrophage surface markers characterizes systemic sclerosis patients with lung involvement
}

Amelia Chiara Trombetta ${ }^{1 \dagger}$, Stefano Soldano ${ }^{1 \dagger}$, Paola Contini ${ }^{2}$, Veronica Tomatis ${ }^{1}$, Barbara Ruaro ${ }^{1}$, Sabrina Paolino ${ }^{1}$, Renata Brizzolara', Paola Montagna ${ }^{1}$, Alberto Sulli ${ }^{1}$, Carmen Pizzorni ${ }^{1}$, Vanessa Smith ${ }^{3,4}$ and Maurizio Cutolo ${ }^{1 *}$ (D)

\begin{abstract}
Background: Systemic sclerosis (SSc) is a disorder characterized by immune system alterations, vasculopathy and fibrosis. SSc-related interstitial lung disease (ILD) represents a common and early complication, being the leading cause of mortality. Monocytes/macrophages seem to have a key role in SSc-related ILD. Interestingly, the classically (M1) and alternatively (M2) activated monocyte/macrophage phenotype categorization is currently under revision.

Our aim was to evaluate if circulating monocyte/macrophage phenotype could be used as biomarker for lung involvement in SSc. To this purpose we developed a wide phenotype characterization of circulating monocyte/macrophage subsets in SSc patients and we evaluated possible relations with lung involvement parameter values.

Methods: A single centre cross-sectional study was performed in fifty-five consecutive SSc patients, during the year 2017. All clinical and instrumental tests requested for SSc follow up and in particular, lung computed tomography (CT) scan, pulmonary function tests (PFTs), Doppler echocardiography with systolic pulmonary artery pressure (SPAP) measurement, blood pro-hormone of brain natriuretic peptide (pro-BNP) evaluation, were performed in each patient in a maximum onemonth period. Flow cytometry characterization of circulating cells belonging to the monocyte/macrophage lineage was performed using specific M1 (CD80, CD86, TLR2 and TLR4) and M2 surface markers (CD204, CD163 and CD206). Nonparametric tests were used for statistical analysis.

Results: A higher percentage of circulating $\mathrm{CD} 204^{+} \mathrm{CD} 163^{+} \mathrm{CD} 206^{+} \mathrm{TLR}^{+} \mathrm{CD} 80^{+} \mathrm{CD} 86^{+}$and $\mathrm{CD} 14^{+} \mathrm{CD} 206^{+} \mathrm{CD} 163^{+} \mathrm{CD}$ $204^{+} \mathrm{TLR}^{+} \mathrm{CD} 80^{+} \mathrm{CD} 86^{+}$mixed M1/M2 monocyte/macrophage subsets, was identified to characterize patients affected by SSc-related ILD and higher systolic pulmonary artery pressure. Mixed M1/M2 monocyte/macrophage subset showed higher percentages in patients positive for anti-topoisomerase antibody, a known lung involvement predictor.

Conclusions: The present study shows for the first time, through a wide flow cytometry surface marker analysis, that higher circulating mixed M1/M2 monocyte/macrophage cell percentages are associated with ILD, SPAP and antitopoisomerase antibody positivity in SSc, opening the path for research on their possible role as pathogenic or biomarker elements for SSc lung involvement.
\end{abstract}

Keywords: Systemic sclerosis, Interstitial lung disease, Pulmonary artery hypertension, Monocyte/macrophage phenotype, M1, M2, Innate immunity, Lung CT scan, Pulmonary function tests, Flow cytometry, Anti-topoisomerase antibody

\footnotetext{
* Correspondence: mcutolo@unige.it

${ }^{+}$Amelia Chiara Trombetta and Stefano Soldano contributed equally to this

work.

${ }^{1}$ Research Laboratory and Academic Division of Clinical Rheumatology,

Department of Internal Medicine, University of Genova, Polyclinic San

Martino Hospital, Genoa, Italy

Full list of author information is available at the end of the article
}

(c) The Author(s). 2018 Open Access This article is distributed under the terms of the Creative Commons Attribution 4.0 International License (http://creativecommons.org/licenses/by/4.0/), which permits unrestricted use, distribution, and reproduction in any medium, provided you give appropriate credit to the original author(s) and the source, provide a link to the Creative Commons license, and indicate if changes were made. The Creative Commons Public Domain Dedication waiver (http://creativecommons.org/publicdomain/zero/1.0/) applies to the data made available in this article, unless otherwise stated. 


\section{Background}

Systemic sclerosis (SSc) is a rare autoimmune disease, characterized by progressive microvascular damage and fibrosis, involving almost all organs of affected patients and predictable by several biomarkers [1].

Interstitial lung disease (ILD) is a common and early complication in SSc patients, and a certain degree of ILD, in the form of non-specific interstitial pneumonia (NSIP), has been shown in $78 \%$ of SSc lung biopsies. Notably, among possible organ involvements in SSc, ILD evolves to the worse prognosis, being the leading cause of mortality in SSc patients [2].

In addition, patients affected by SSc-associated ILD have a high risk to develop cardiopulmonary disease and pulmonary hypertension. After pulmonary hypertension development, severe impairments in both physical and emotional domains of health-related quality of life were demonstrated [3]. The 3-year death rate in SSc patients affected by pulmonary hypertension was calculated to be $44-64 \%[4,5]$.

SSc-associated ILD was demonstrated to be early recognized by lung computed tomography (CT) scan. On the other hand, the wide range of pulmonary function test (PFT) normal values ( $80-120 \%$ of predicted) may determine its reduced sensitivity [6].

Several studies recently highlighted the genetic and epigenetic aberrations involved in the SSc pathogenesis $[7,8]$. Importantly, major gene signatures related to phenotype, activation and migration of macrophages demonstrated to be relevant to the progressive pulmonary fibrosis, indicating macrophages as key players $[9,10]$.

Intriguingly, imbalance in macrophage phenotype features and macrophage activation have been lately considered essential for the development of inflammatory-autoimmune, fibrotic, infective and neoplastic disorders characterized by lung involvement [11-16]. Macrophages have been initially categorized as classically (M1) or alternatively activated (M2), mirroring T cells categories. M1 macrophages express specific phenotype markers, including toll-like receptors (i.e., TLR2 and TLR4) and the co-stimulatory molecules CD80 and CD86, and are involved in triggering intensive inflammation and tissue damage [17]. M2 macrophages primarily express the mannose receptor-1 (CD206) and macrophage scavenger receptors (CD204 and CD163), and they are associated with $\mathrm{T}$ helper (Th) 2 response, tissue repair and fibrosis $[18,19]$.

Recently, classifications based on a wider spectrum of phenotypes of which M1 and M2 subsets would constitute the two extremes have been described [20].

Moreover, it was observed that the majority of alveolar macrophages combine M1 and M2 features in steady state and that the mixed M1/M2 phenotype can be altered by HIV infection [21].
Interestingly, a recent preliminary study demonstrated higher percentages of circulating mixed M1/M2 monocytes/macrophages in SSc patients compared to healthy subjects (HSs) [22]. The aim of the present study was to effectuate a wide phenotype characterization of circulating monocytes/macrophages in consecutive SSc patients stratified according to the severity of lung and right heart involvement, through lung CT scan imaging, PFTs, pro-BNP blood values, and Doppler echocardiography.

\section{Methods \\ Study design}

As part of the regular follow up approved by international guidelines for SSc, all patients underwent clinical examination and instrumental exams over a period of time of up to one month. In particular, lung CT scan, PFT with diffusing capacity of the lungs for carbon monoxide (DLCO), Doppler echocardiography with systolic pulmonary artery pressure (sPAP) measurement, pro-hormone of brain natriuretic peptide (pro-BNP) blood values, were performed for lung-right heart involvement evaluation. The assumption of medications was also considered.

The study was approved by the Ethics Committee of Polyclinic San Martino Hospital, Genoa, Italy (protocol number: 273-reg-2015).

Throughout the manuscript, the investigated cells were defined as circulating "monocytes/macrophages". This is because we wanted to use as much surface markers as possible, including those expressed from mature and polarized macrophages, to better restrict the investigation to monocytes and macrophages, and to study the mixed M1/ M2 phenotype, independently from the cell maturation state. In fact, we did not want to exclude also a possible presence of circulating cells in later maturation stages. Therefore, we used different gating strategies, as reported in Additional file 1, including typical markers of immature and mature cells, as previously described [22].

\section{Participants}

Fifty-five consecutive SSc patients (50 females and 5 males, mean age $63 \pm 13$ years), undergoing complete disease staging in a day hospital setting at the Rheumatology Division of Genoa University, were enrolled in the study after written informed consent. Among the enrolled SSc patients, 36 were characterized by a limited cutaneous $(\mathrm{lcSSc})$ disease form and 19 were characterized by a diffused cutaneous ( $\mathrm{dcSSc}$ ) disease form. SSc diagnosis was done according to the American College of Rheumatology (ACR)/European League Against Rheumatism (EULAR) 2013 criteria [23, 24].

Data from blood samples derived from a population of 27 sex and gender matched HSs, analysed in a recent preliminary study, were applied here for comparison with SSc patients, for the most significant results [22]. 


\section{Lung and right heart involvement parameters}

Lung CT scan, PFTs and sPAP, pro-BNP value measurements were performed in each patient in the same period of the other examinations scheduled for SSc follow-up. Results were interpreted by the same operator for each type of diagnostic test.

Afterwards, patients were stratified according to the presence or the absence of any interstitial involvement at lung CT scan (SSc-ILD group versus SSc No-ILD group, respectively). Therefore, patients were stratified also according to the presence or the absence of single CT scan abnormalities, characteristically described in SSc lung involvement: ground glass opacities (defined as an area of increased attenuation in the absence of architectural distortion) of lower lobes, ground glass opacities of upper lobes, peripheral septal thickening, apical fibrotic (architectural distortion with reticular intra-lobular interstitial thickening) changes, diffused fibrotic changes, traction bronchiectasis and bronchiolectasis (dilatation of the airways in the peripheral portion of the lung) and enlarged mediastinal nodes [25].

As regards PFT, forced vital capacity (FVC), DLCO and $\mathrm{FVC/DLCO}$ ratio values were reported and analyzed for each SSc patient. In agreement with previous studies, the FVC/DLCO ratio higher than 1.5 was considered suggestive for pulmonary vasculopathy in SSc patients [26].

\section{Flow cytometry}

After enrolment, peripheral blood was collected in a lithium-heparin single tube from each SSc patient.

To identify monocyte/macrophage lineage surface markers CD14-APC-Vio770 and CD45-VioGreen antibodies were used. The characterization of M2 phenotype was performed using CD204-PE, CD163-PE-Vio770 and CD206-PeerCP-Vio700, whereas the M1 phenotype was investigated using CD80-APC, CD86-VioBlue, TLR2-PEVio615 and TLR4-VioBright-FITC antibodies. CD66b -FITC was used to identify and exclude granulocytes (Miltenyi Biothech, Bergisch Gladbach, Germany).

A total of $0.1 \mathrm{ml}$ of peripheral blood was incubated with $10 \mu \mathrm{l}$ of antibody for $15 \mathrm{~min}$ at room temperature, then erythrocytes were lysed and leucocytes post-fixed. Afterwards, the flow cytometry analysis was performed.

Three initial gating strategies were implemented to investigate circulating monocyte/macrophage phenotype over total leucocyte population and included in the Additional file 1 . The first initial gating strategy evaluated the $\mathrm{CD} 14^{+}$cells over total leucocyte population. In this $\mathrm{CD}_{14}{ }^{+}$cell population, circulating monocytes/macrophages showing an M2 phenotype were characterized based on the expression of CD204, CD163 and CD206. Therefore, a second initial gating strategy evaluated the $\mathrm{CD}^{2} 4^{+}$cells in the leucocyte population, excluding lymphocytes, $\mathrm{CD} 66 \mathrm{~b}^{+}$granulocytes, doublets and cellular debris. In the $\mathrm{CD}_{204}{ }^{+}$population, circulating cells co-expressing CD163 and CD206 were detected to characterize monocytes/macrophages showing an M2 phenotype. Cells positive for M2 phenotype markers (CD204, CD163, CD206) and M1 phenotype markers (TLR4, CD80 and CD86) were investigated to identify the presence of cells with a mixed M1/M2 phenotype, as recently reported [22]. Although lymphocytes and neutrophils are excluded in the initial gating strategy starting from CD204 ${ }^{+}$cells, no specific dendritic cell markers were investigated to discriminate these cells and then they might be probably present in a limited percentage in the M1/M2 mixed population.

Finally, a third initial gating strategy was made up to detect monocytes/macrophages showing prominently M1 surface markers CD80, CD86, TLR2 and TLR4 [27].

Flow cytometric analysis was performed using a Navios Flow Cytometer and the Kaluza analysis software (Beckman Coulter, Milan, Italy), evaluating a total of $5 \times$ $10^{6}$ cells and detecting more than 30 events in the smallest subset investigated, according to consensus guidelines on the minimal residual disease [28].

\section{Statistical analysis}

Data were analyzed using IBM SPSS Statistics Version 21.0. (IBM Corp: Armonk, NY). Non-parametric tests were applied for statistical analysis and in particular Mann-Whitney U test was used for comparing data with an ordinal distribution between two independent groups. Kruskal-Wallis test was chosen to assess significantly different distributions of continuous dependent variables by a categorical independent variable with more than two groups. Finally, bivariate Pearson's correlation was calculated to measure linear relationship between two variables with ordinal distribution. A $p$-value lower than 0.05 was considered as statistically significant. The results were expressed as median \pm standard deviation (SD) and graphically represented through box and whisker plots.

\section{Results}

Demographics and clinical parameters are summarized in Table 1.

Only 5 males were enrolled among the SSc patient population, consequently the sex variable was not used for the analysis.

\section{Associations between auto-antibody positivity, pro-BNP blood values, disease form, and monocyte/macrophage phenotype}

Anti-topoisomerase antibody (Anti-Scl70) positivity was associated with lower $\mathrm{FVC} \%$ (Scl70 $+=84.5 \pm 14 \%$ vs. Scl70- $=112.8 \pm 22 ; \quad p<0.0001)$ and higher pro-BNP values $(\mathrm{Scl} 70+=790 \pm 883$ vs. Scl $70-=213 \pm 243 p=$ 0.01) (Fig. 1a and b). 
Table 1 Demographic clinical and imaging data from the whole systemic sclerosis patient population

\begin{tabular}{|c|c|}
\hline $\begin{array}{l}\text { Demographic, clinical and } \\
\text { imaging data in SSC PTs }\end{array}$ & $\begin{array}{l}\text { Mean } \pm \text { SD OR } \\
\text { number-percentage }\end{array}$ \\
\hline Age (years, mean $\pm S D$ ) & $63 \pm 13$ \\
\hline Sex (females/males) & $50 / 5$ \\
\hline RP duration (years, mean \pm SD) & $5.8 \pm 10$ \\
\hline SSc duration (years, mean \pm SD) & $8.4 \pm 6$ \\
\hline SSc form $=$ LCSSc/dcSSC $(n=\%)$ & $\begin{array}{l}36=65.5 \% / \\
19=34.5 \%\end{array}$ \\
\hline ANA $(n=\%)$ & $55=100 \%$ \\
\hline $\operatorname{ACA}(n=\%)$ & $20=36.4 \%$ \\
\hline Anti-Scl-70 Ab ( $n=\%)$ & $23=41.8 \%$ \\
\hline ILD at CT scan ( $n=\%)$ & $37=67.3 \%$ \\
\hline Ground glass opacities, lower lobes $(n=\%)$ & $13=23.6 \%$ \\
\hline Ground glass opacities, upper lobes $(n=\%)$ & $8=14.5 \%$ \\
\hline $\begin{array}{l}\text { Ground glass opacities, upper and lower } \\
\text { lobes }(n=\%)\end{array}$ & $8=14.5 \%$ \\
\hline Peripheral septal thickening $(n=\%)$ & $31=56.4 \%$ \\
\hline Apical fibrotic changes ( $n=\%)$ & $20=36.4 \%$ \\
\hline Diffused fibrotic changes $(n=\%)$ & $15=27.3 \%$ \\
\hline Enlarged mediastinal nodes $(n=\%)$ & $16=29.1 \%$ \\
\hline $\begin{array}{l}\text { Traction bronchiectasis and bronchiolectasis } \\
(n=\%)\end{array}$ & $16=29.1 \%$ \\
\hline FVC\% (mean \pm SD) & $104 \pm 24$ \\
\hline DLCONA\% (mean \pm SD) & $71.5 \pm 20$ \\
\hline sPAP mmHg (mean \pm SD) & $34 \pm 7$ \\
\hline Pro-BNP (pg/ml, mean $\pm \mathrm{SD})$ & $1423 \pm 5119$ \\
\hline On immunosuppressive therapy $(n=\%)$ & $32=56.1 \%$ \\
\hline On glucocorticoids $(n=\%)$ & $9=16.4 \%$ \\
\hline On ERAs $(n=\%)$ & $16=28.1 \%$ \\
\hline
\end{tabular}

Data are expressed as means \pm standard deviations or numbers $=$ percentages of the total population. SSC Systemic sclerosis, PTs patients, RP Raynaud's phenomenon, $S D$ standard deviation, ILD interstitial lung disease, $A N A$ Anti-nuclear antibody, ACA: Anti-centromere antibodies, Ab anti-Scl70 anti-topoisomerase antibody, CT computed tomography, FVC forced vital capacity, DLCO diffusing capacity of the lungs for carbon monoxide, SPAP systolic pulmonary artery pressure, pro-BNP prohormone of brain natriuretic peptide, ERAs Endothelin 1 receptor antagonists. No other vasomodulating therapies were used by the selected SSC patients

In the circulating $\mathrm{CD}^{204^{+}}$cell population of Scl70 positive SSc patients, by Mann-Whitney test, several mixed M1/M2 macrophage subsets showed higher percentages compared to Scl70 negative SSc patients: $\mathrm{CD} 204^{+} \mathrm{CD} 163$ ${ }^{+} \mathrm{CD}_{206}{ }^{+} \mathrm{TLR}^{+}{ }^{+} \mathrm{CD} 14^{-}$cells (Scl70+ $=2.4 \pm 4.6 \%$, vs. Scl70$=0.64 \pm 7.9 \%, \quad p=0.036), \quad \mathrm{CD} 204^{+} \mathrm{CD} 163^{+} \mathrm{CD} 206^{+} \mathrm{TLR} 4$ ${ }^{+} \mathrm{CD}^{+}{ }^{+}$cells $(\mathrm{Scl} 70+=8.2 \pm 8.2 \%$ vs. $\mathrm{Scl} 70-=0.86 \pm 4.4 \%, p$ $=0.027$, Fig. 1e), CD204 ${ }^{+} \mathrm{CD} 163^{+} \mathrm{CD} 206^{+} \mathrm{TLR}^{+} \mathrm{CD}^{+} 6^{+}$cells $(\mathrm{Scl} 70+=2.2 \pm 6.9 \% \quad$ vs. $\quad \mathrm{Scl} 70-=0.7 \pm 3.6 \%, \quad p=0.046)$, $\mathrm{CD} 204^{+} \mathrm{CD} 163^{+} \mathrm{CD} 206^{+} \mathrm{TLR}^{+} \mathrm{CD} 80^{+} \mathrm{CD}^{+} 6^{+} \quad$ cells $(\mathrm{Scl} 70+=1.6 \pm 6.7 \%$ vs. Scl70- $=0.54 \pm 3.6 \%, p=0.036$, Fig. 1c, d, f).
Using HS data, as reported in the Methods section, Kruskal-Wallis test was performed and significantly lower percentages for the same cell populations in HSs were obtained (HSs values for $\mathrm{CD} 204^{+} \mathrm{CD} 163^{+} \mathrm{CD} 206^{+} \mathrm{TLR}^{+} \mathrm{CD} 14^{-}$ cells $=0.17 \pm 0.41 \%, p<0.0001$ vs. SSc patients, pairwise comparison: HSs vs Scl70- $p=0.05$, HSs vs Scl70+ $p<$ $0.0001 ; \mathrm{CD}_{204}{ }^{+} \mathrm{CD}_{163}{ }^{+} \mathrm{CD} 206^{+} \mathrm{TLR}^{+}{ }^{+} \mathrm{CD} 80^{+}$cells $=0.16 \pm$ $0.48 \%, p<0.0001$ vs. SSc patients, pairwise comparison: HSs vs Scl70- $p=0.001, \quad \mathrm{HSs}$ vs $\mathrm{Scl} 70+p<0.0001$; $\mathrm{CD}_{204}{ }^{+} \mathrm{CD} 163^{+} \mathrm{CD}^{206}{ }^{+} \mathrm{TLR}^{+}{ }^{+} \mathrm{CD} 86^{+}$cells $=0.18 \pm 0.36 \%, p$ $<0.0001$ SSc patients, pairwise comparison: HSs vs Scl70- $p$ $=0.001, \quad \mathrm{HSs} \quad$ vs Scl70+ $p<0.0001 ; \quad \mathrm{CD} 204^{+} \mathrm{CD} 163$ ${ }^{+} \mathrm{CD} 206{ }^{+} \mathrm{TLR}^{+}{ }^{+} \mathrm{CD} 80^{+} \mathrm{CD}^{+} 6^{+}$cells $=0.08 \pm 0.29 \%, p<0.0001$ SSc patients, pairwise comparison: HSs vs Scl70- $p=0.001$, HSs vs $\mathrm{Scl} 70+p<0.0001$, Additional file 2).

No association was reported between Scl70 Ab positivity and cells expressing exclusively M1 or M2 phenotype markers.

Anti-centromere antibodies (ACA) positivity was associated with older age at the time of the study (ACA+ = $70 \pm 7$ vs. $\mathrm{ACA}-=57 \pm 14$ years, $p=0.002$ ), longer SSc duration $(\mathrm{ACA}+=10 \pm 6$ vs. $\mathrm{ACA}-=7 \pm 6$ years, $p=$ 0.049), higher FVC percentage $(\mathrm{ACA}+=118 \pm 19 \%$ vs. ACA- $=94 \pm 21 \%, p<0.0001)$, and lower gammaglobulin percentage values $(\mathrm{ACA}+=14.8 \pm 3 \%$ vs. $\mathrm{ACA}-=17.3 \pm$ $3 \%, p=0.004)$.

ACA positive patients showed a higher percentage of CD $14^{+}$cells $(\mathrm{ACA}+=7.3 \pm 1.9 \%$ vs. ACA $-6.3 \pm 2.4 \%, p$ $=0.022$ ). Several cells more clearly polarized towards an M1 or M2 phenotype segregated with ACA positivity: $\mathrm{CD}_{14}{ }^{+} \mathrm{TLR}^{+}(\mathrm{ACA}+=7.2 \pm 2.6 \%$ vs. $\mathrm{ACA}-=6 \pm 3 \%, p$ $=0.046), \mathrm{CD}_{14}{ }^{+} \mathrm{CD} 163^{+}(\mathrm{ACA}+=7 \pm 1.9 \%$ vs. $\mathrm{ACA}-=$ $5.8 \pm 2.6 \%, p=0.025)$, and $\mathrm{CD} 14^{+} \mathrm{CD} 204^{+} \mathrm{CD} 163^{+}(\mathrm{ACA}$ $+=0.18 \pm 0.4 \%$ vs. ACA - $=0.08 \pm 0.3 \%, p=0.011)$.

Only CD $204^{+} \mathrm{CD} 163^{+} \mathrm{CD} 206^{+} \mathrm{TLR}_{4}{ }^{+} \mathrm{CD} 80^{+}$cells, in the circulating $\mathrm{CD}_{20} 4^{+}$cells, showed higher percentages in dcSSc compared to lcSSc patients $(\mathrm{dcSSc}=2.96 \pm 8 \%$ vs. $\mathrm{lcSSc}=0.94 \pm 5 \%, p=0.047)$. The Kruskal-Wallis test, executed adding $\mathrm{HSs}$ data, showed significantly lower CD $204^{+} \mathrm{CD}_{163}{ }^{+} \mathrm{CD}_{206}{ }^{+} \mathrm{TLR}_{4}{ }^{+} \mathrm{CD} 80^{+}$cell percentages in comparison to both lcSSc and dcSSc $(0.16 \pm 0.48 \%, p<$ 0.0001 , globally and after pairwise comparison).

\section{Associations between lung disease evaluated at CT scan and monocyte/macrophage phenotype}

The only gating strategy that effectively highlighted differences in circulating monocyte/macrophage phenotype between SSc-ILD versus SSc-No ILD group was the one based on initial gating of CD204 ${ }^{+}$ cells (Table 2).

No significant difference was reported for total CD204 ${ }^{+}$cell percentage, over circulating leukocytes, between SSc-ILD patient and SSc-No ILD patient groups (Table 2). 
A

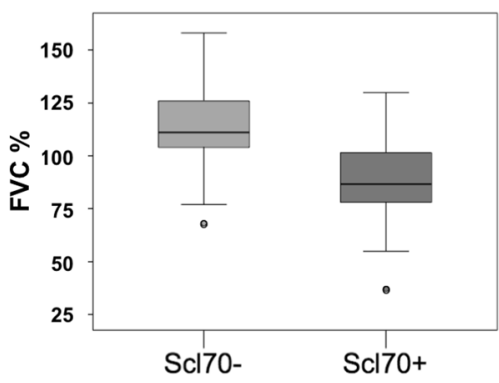

C

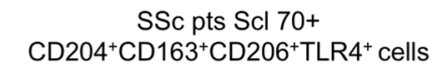

B

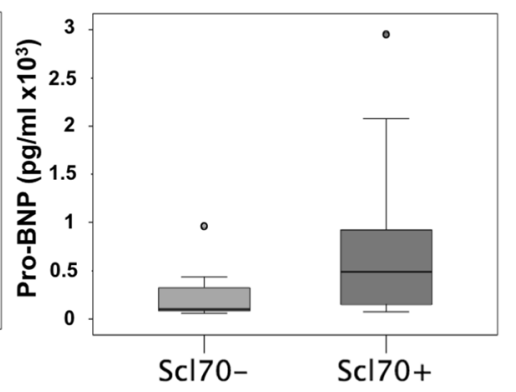

D CD204+CD $163^{+}$CD206 ${ }^{+}$TLR4 ${ }^{+}$cells

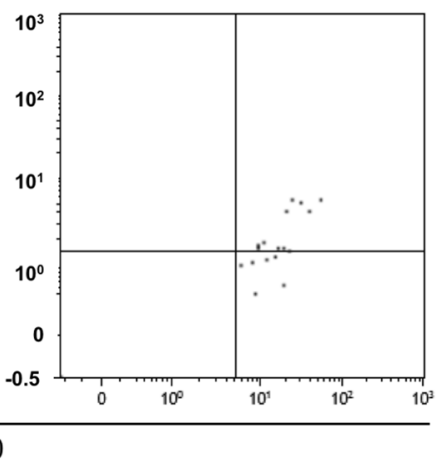

$\mathrm{F}$
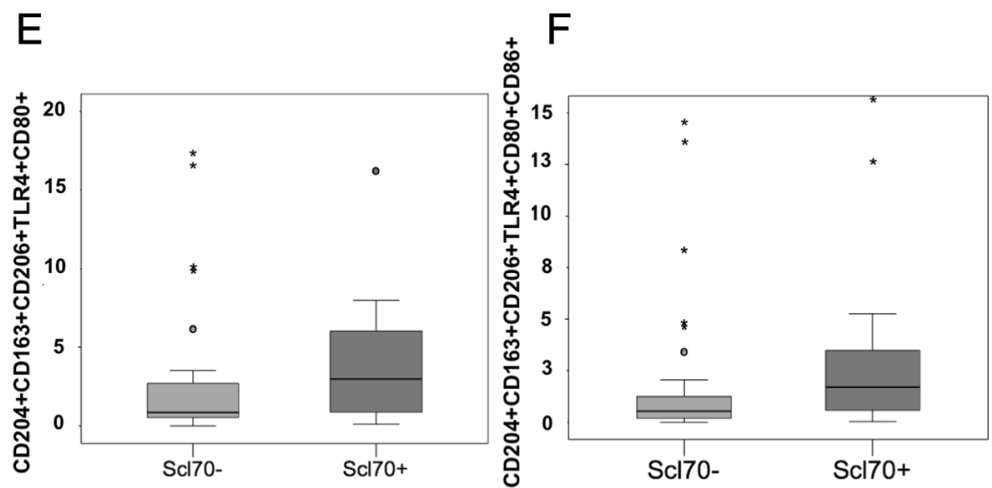

Fig. 1 Ab anti Scl70 positivity: associations with FVC\%, Pro-BNP blood values and mixed M1/M2 cells percentages. a and $\mathbf{b}$, clinical associations of AntiScl70 Ab positivity with lower FVC\% and higher pro-BNP values are shown. $\mathbf{c}$ and $\mathbf{d}$ show the representative dot plots from the flow cytometry analysis of the mixed M1/M2 CD204 ${ }^{+} \mathrm{CD} 163^{+} \mathrm{CD} 206^{+} \mathrm{TLR}^{+} \mathrm{CD} 80^{+} \mathrm{CD} 86^{+}$cell subset is shown in patients with positive and negative Ab anti-Scl70. Significant differences $\left(p=0.027\right.$ ) are shown between average percentages of circulating mixed M1/M2 subset $C D 204^{+} C D 163^{+} C D 206^{+} T L R 4^{+} C D 80^{+}$over total $\mathrm{CD}^{204^{+}}$cells, in Scl70+ vs Scl70- patients (e) and between percentage of circulating mixed M1/M2 subset CD204 ${ }^{+} \mathrm{CD} 163^{+} \mathrm{CD} 206^{+} \mathrm{TLR}^{+} \mathrm{CD} 80^{+} \mathrm{CD} 86^{+}$ over total CD204 ${ }^{+}$cells, in Scl70+ vs Scl70- patients (f). Anti-Scl70 = Anti-topoisomerase; FVC = forced vital capacity; pro-BNP = prohormone of brain natriuretic peptide

Considering the $\mathrm{CD}_{20} 4^{+}$cell population, SSc-ILD patients showed a significant increased percentage of circulating $\mathrm{CD} 204^{+} \mathrm{CD} 163^{+}$cells compared to SSc-No ILD patients (Table 2). Likewise, circulating CD204 ${ }^{+} \mathrm{CD} 163^{+} \mathrm{TLR}^{+}$cells, CD204 ${ }^{+} \mathrm{CD}_{163}{ }^{+} \mathrm{CD} 206^{+} \mathrm{TLR}^{+}$cells, showed significant higher percentages in the SSc-ILD group (Table 2). Among $\mathrm{CD} 04^{+} \mathrm{CD} 163^{+} \mathrm{TLR}^{+} \mathrm{CD} 206^{+}$cells, only $\mathrm{CD} 14^{-}$and not $\mathrm{CD}_{14}{ }^{+}$cells showed significantly higher percentages in the SSc-ILD group (Table 2).
Remarkably, in the $\mathrm{CD} 204^{+} \mathrm{CD} 163^{+} \mathrm{CD} 206^{+} \mathrm{TLR}^{+}$cell population, mixed M1/M2 phenotype cells expressing CD80 and CD86 markers resulted significantly increased in the SSc-ILD group compared to the SSc-No ILD group (Fig. 2 a, b, c, d and Table 2).

No differences were observed between SSc-ILD and SSc-No ILD patients in the percentage of total circulating CD14 ${ }^{+}$cells $(6.68 \pm 1.8 \%$ and $7.57 \pm 2.5 \%$, respectively, $p=0.18$ ). 
Table 2 The CD204 positive cell population percentages are shown in patients with (SSc-ILD) or without interstitial lung disease (SSC-No ILD) at lung CT scan and healthy subjects (HSS)

\begin{tabular}{|c|c|c|c|c|c|}
\hline Analysis of circulating CD204+ cells & SSc-ILD (37) & SSc-No-ILD (18) & $\mathrm{p}(\mathrm{MW})$ & HSs (27) & $p(K W)$ \\
\hline$\overline{\mathrm{CD}_{20} 4^{+}(\%)}$ & $0.5 \pm 0.40$ & $0.8 \pm 0.7$ & $p=0.13$ & $0.7 \pm 0.3$ & 0.21 \\
\hline $\mathrm{CD} 204^{+} \mathrm{CD} 163^{+}$(\%leukocytes) & $0.08 \pm 0.22$ & $0.09 \pm 0.14$ & $p=0.65$ & $0.03 \pm 0.03$ & 0.001 \\
\hline 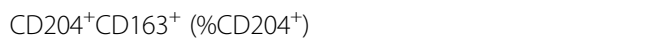 & $13.7 \pm 15$ & $8.4 \pm 13$ & $p=0.034$ & $6.3 \pm 3$ & $<0.0001$ \\
\hline $\mathrm{CD} 04^{+} \mathrm{CD} 163^{+} \mathrm{TLR}^{+}$(\%leukocytes) & $0.03 \pm 0.22$ & $0.02 \pm 0.15$ & $p=0.34$ & $0.008 \pm 0.01$ & $<0.0001$ \\
\hline 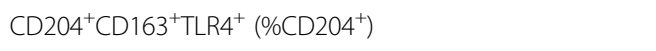 & $6.2 \pm 16$ & $2.9 \pm 15$ & $p=0.025$ & $1.4 \pm 1.7$ & $<0.0001$ \\
\hline $\mathrm{CD} 04^{+} \mathrm{CD} 163^{+} \mathrm{CD} 206^{+}$(\%leukocytes) & $0.01 \pm 0.1$ & $0.01 \pm 0.05$ & $p=0.65$ & $0.008 \pm 0.01$ & 0.001 \\
\hline $\mathrm{CD} 204^{+} \mathrm{CD} 163^{+} \mathrm{CD} 206^{+}\left(\% \mathrm{CD} 204^{+}\right)$ & $4 \pm 7.4$ & $1.9 \pm 5.6$ & $p=0.07$ & $1.1 \pm 1.2$ & $<0.0001$ \\
\hline $\mathrm{CD} 04^{+} \mathrm{CD} 163^{+} \mathrm{CD}^{2} 06^{+} \mathrm{TLR}^{+}$(\%leukocytes) & $0.014 \pm 0.1$ & $0.11 \pm 0.05$ & $p=0.20$ & $0.003 \pm 0.004$ & $<0.0001$ \\
\hline 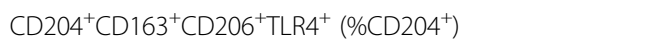 & $2.7 \pm 7.3$ & $1.1 \pm 5.9$ & $p=0.013$ & $0.5 \pm 0.6$ & $<0.0001$ \\
\hline $\mathrm{CD} 04^{+} \mathrm{CD}_{163}{ }^{+} \mathrm{CD} 206^{+} \mathrm{TLR}^{+} \mathrm{CD} 14^{+}$(\%leukocytes) & $0.003 \pm 0.014$ & $0.004 \pm 0.003$ & $p=0.795$ & $0.001 \pm 0.003$ & 0.008 \\
\hline 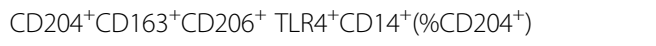 & $0.73 \pm 1.4$ & $0.27 \pm 0.54$ & $p=0.097$ & $0.20 \pm 0.38$ & $<0.0001$ \\
\hline 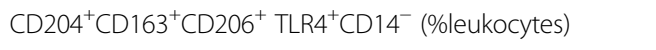 & $0.009 \pm 0.08$ & $0.006 \pm 0.05$ & $p=0.092$ & $0.001 \pm 0.002$ & $<0.0001$ \\
\hline 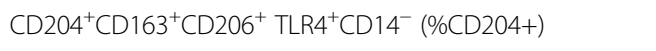 & $1.93 \pm 6.56$ & $0.6 \pm 5.69$ & $p=0.029$ & $0.17 \pm 0.41$ & $<0.0001$ \\
\hline 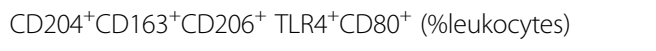 & $0.01 \pm 0.09$ & $0.004 \pm 0.05$ & $p=0.041$ & $0.001 \pm 0.003$ & $<0.0001$ \\
\hline 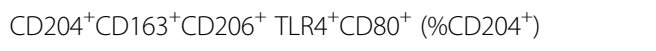 & $2.07 \pm 6.83$ & $0.5 \pm 5.33$ & $p=0.010$ & $0.16 \pm 0.48$ & $<0.0001$ \\
\hline $\mathrm{CD} 24^{+} \mathrm{CD} 163^{+} \mathrm{CD} 206^{+} \mathrm{TLR}^{+} \mathrm{CD} 6^{+}$(\%leukocytes) & $0.008 \pm 0.08$ & $0.005 \pm 0.04$ & $p=0.082$ & $0.001 \pm 0.002$ & $<0.0001$ \\
\hline 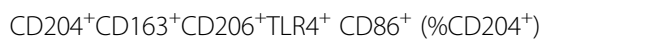 & $1.16 \pm 5.8$ & $0.72 \pm 4.1$ & $p=0.023$ & $0.19 \pm 0.36$ & $<0.0001$ \\
\hline $\mathrm{CD} 204^{+} \mathrm{CD} 163^{+} \mathrm{CD} 206^{+} \mathrm{TLR} 4^{+} \mathrm{CD} 80^{+} \mathrm{CD}^{+} 6^{+}$(\%leukocytes) & $0.04 \pm 0.08$ & $0.002 \pm 0.03$ & $p=0.036$ & $0.0006 \pm 0.001$ & $<0.0001$ \\
\hline 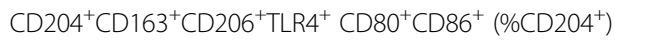 & $1 \pm 5.6$ & $0.39 \pm 4$ & $p=0.021$ & $0.08 \pm 0.2$ & $<0.0001$ \\
\hline
\end{tabular}

By Mann-Whitney test, several mixed M1/M2 cell populations were found to show significantly higher percentages ( $p$ MW highlighted in bold) in SSc patients affected by ILD, compared to SSc patients with no ILD. On the right, Kruskal-Wallis test was performed adding HSs data, obtaining more significant results ( $p$ KW)

No differences were observed between SSc-ILD and SSc-No ILD patients in the percentage of circulating monocytes/macrophages expressing only surface markers considered to be M1 specific.

\section{Monocytes/macrophages phenotype and single CT scan alterations associated with interstitial lung disease, in SSC patients}

Interestingly, SSc patients showing fibrotic changes diffused to upper and lower lobes at lung CT scan, seemed to be characterized by a slightly higher percentage of mixed M1/M2 monocytes/macrophages and characterized as $\mathrm{CD} 14^{+} \mathrm{CD} 206^{+} \mathrm{CD} 163^{+} \mathrm{CD} 80^{+} \mathrm{CD} 86^{+}$compared to patients with less or no lung fibrosis $(0.001 \pm 0.008$ and $0.0006 \pm 0.006, p=0.044)$. Coherently, the same cell population showed higher percentages in patients presenting bronchiectasis or bronchiolectasis $(0.002 \pm$ $0.008 \%$ and $0.0006 \pm 0.006 \%, p=0.021$ ).

No significant difference was reported in circulating monocyte/macrophage phenotype between patients with reported ground glass opacities localized at lower or upper lung lobes or diffused to both locations at lung CT scan. Similarly, no significant difference was observed in SSc patients for whom peripheral septal thickening, apical fibrotic changes, or enlarged mediastinal nodes were reported at lung CT scan.
Correlations between circulating monocyte/macrophage phenotype, PFTs and SPAP values, in SSc patients

Higher percentages of circulating mixed M1/M2 monocyte/macrophage subset, characterized as $\mathrm{CD}_{14}{ }^{+} \mathrm{CD} 206^{+} \mathrm{CD} 163^{+} \mathrm{CD} 204^{+} \mathrm{TLR}_{4}{ }^{+} \mathrm{CD} 80^{+} \mathrm{CD} 86^{+}$

cells, showed a weak linear negative correlation with DLCO\% ( $p=0.046, r=-0.28$, Fig. 3a).

No linear correlations were observed between macrophage subsets phenotype and $\mathrm{FVC} \%$ values.

A FVC/DLCO ratio higher than 1.5 was associated with several circulating monocyte/macrophage subset percentages, and in particular: $\mathrm{CD} 204^{+} \mathrm{CD} 163^{+}$cells calculated over total $\mathrm{CD} 204^{+}$cells $(\mathrm{FVC} / \mathrm{DLCO}<1.5=9 \pm$ $15 \%$ vs. FVC/DLCO $>1.5=13 \pm 14 \%, p=0.006), \mathrm{CD}^{2} 24^{+}$ $\mathrm{CD} 163^{+} \mathrm{TLR}^{+}{ }^{+}$cells calculated over CD204 ${ }^{+}$cells $(\mathrm{FVC} /$ $\mathrm{DLCO}<1.5=3 \pm 15 \%$ vs. FVC/DLCO $>1.5=6.4 \pm 15 \%, p$ $=0.025), \mathrm{CD}_{20} 4^{+} \mathrm{CD} 163^{+} \mathrm{TLR}^{+}$cells calculated over total leucocytes (FVC/DLCO $<1.5=0.02 \pm 0.25 \%$, vs. $\mathrm{FVC} / \mathrm{DLCO}>1.5=0.04 \pm 0.02 \%, \quad p=0.039), \quad \mathrm{CD} 204^{+}$ $\mathrm{CD} 163^{+} \mathrm{CD} 206^{+} \mathrm{TLR}^{+}{ }^{+} \mathrm{CD} 86^{+}$cells calculated over total leucocytes (FVC/DLCO $<1.5=0.008 \pm 0.03$ vs. FVC/ DLCO $>1.5=0.04 \pm 0.09 \% \%, p=0.041$, Fig. 3b). As regards cell subsets calculated over total $\mathrm{CD} 14^{+}$cells, significant differences were observed with an $\mathrm{FVC} /$ DLCO ratio lower or higher than 1.5: $\mathrm{CD} 14^{+} \mathrm{CD} 163^{+}$ cells $(\mathrm{FVC} / \mathrm{DLCO}<1.5=5.8 \pm 2.4 \%$ vs. $\mathrm{FVC} / \mathrm{DLCO}>1.5$ 


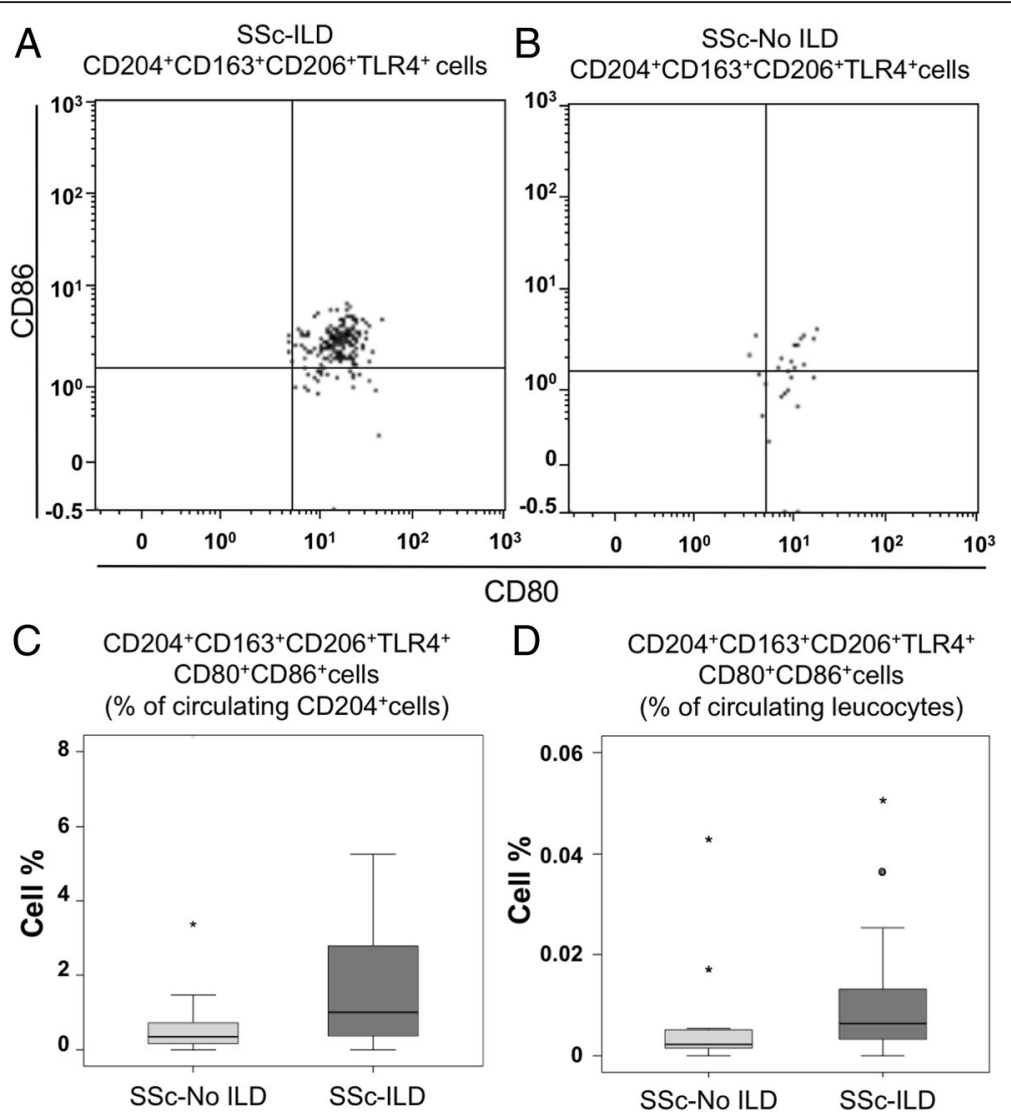

Fig. 2 LLD affected SSc patients: associations with mixed M1 M2 cells percentage. a and $\mathbf{b}$, representative dot plots from the flow cytometry analysis of the CD204 + CD163 + CD206 + TLR4 + CD80 + CD86+ cell subset in SSc patients affected by ILD and not affected by ILD are shown. Mixed M1/M2 cells expressing CD80 and CD86 markers, among CD204 ${ }^{+} C D 163^{+} \mathrm{TLR}^{+} \mathrm{CD} 206^{+}$cells, resulted significantly increased in percentage in the SSc-ILD group compared to the SSc-No ILD group, if calculated both over total CD204 ${ }^{+}$cells (c) and over total circulating leukocytes (d)

$=6.9 \pm 2.3 \%, \quad p=0.044), \quad \mathrm{CD}_{14}{ }^{+} \mathrm{CD} 206^{+}$cells $(\mathrm{FVC} /$ $\mathrm{DLCO}<1.5=6.1 \pm 2.8 \%$ vs. FVC $/ \mathrm{DLCO}>1.5=7.2 \pm 2.8 \%$, $p=0.05), \mathrm{CD}_{14}{ }^{+} \mathrm{CD} 206^{+} \mathrm{CD} 163^{+}$cells $(\mathrm{FVC} / \mathrm{DLCO}<1.5$ $=5.9 \pm 2.7 \%$ vs. FVC/DLCO $>1.5=6.8 \pm 2.7 \%, p=0.046)$, $\mathrm{CD} 14^{+} \mathrm{CD}_{206}{ }^{+} \mathrm{CD} 163^{+} \mathrm{CD} 86^{+}$cells $(\mathrm{FVC} / \mathrm{DLCO}<1.5=$ $0.01 \pm 0.022 \%$ vs. FVC/DLCO $>1.5=0.03 \pm 0.03 \%, p=$ 0.034, Fig. 3c), and $\mathrm{CD} 14^{+} \mathrm{CD} 206^{+} \mathrm{CD} 163^{+} \mathrm{CD} 204^{+}$ $\mathrm{TLR}^{+}{ }^{+} \mathrm{CD} 80^{+} \mathrm{CD} 6^{+}$cells $(\mathrm{FVC} / \mathrm{DLCO}<1.5=0.0001 \pm$ $0.0005 \%$ vs. FVC/DLCO $>1.5=0.001 \pm 0.005 \% ; p=0.005$, Fig. 3d, Additional file 3). Moreover, the higher percentage of mixed $\mathrm{M} 1 / \mathrm{M} 2 \mathrm{CD}_{1} 4^{+} \mathrm{CD} 206^{+} \mathrm{CD} 163^{+} \mathrm{CD} 204^{+}$ $\mathrm{TLR}^{+} \mathrm{CD}{ }^{+} \mathrm{CD}^{+} 6^{+}$cell subset correlated positively with the sPAP value $(p=0.028, r=0.29$, Fig. $3 \mathrm{e})$.

\section{Discussion}

The results of the present study demonstrated that a population of circulating cells belonging to the monocyte/ macrophage lineage and expressing surface markers of both M1 and M2 phenotypes exists, in significantly high percentages, in SSc patients diagnosed as affected by ILD at CT scan. Additionally, higher percentages of mixed M1/M2 circulating monocytes/macrophages resulted linearly correlated with lower values of DLCO\%, with an FVC/DLCO ratio higher then 1.5, and with higher PAPs values. Finally, mixed M1/M2 cell populations demonstrated to be associated with positivity for Scl-70 antibody, a well-known predictor for lung function decline, and less strictly with diffused disease form [29, 30].

Of note, among the circulating leucocyte population, two initial gating strategies moving from CD204 ${ }^{+}$cells and $\mathrm{CD}_{14}{ }^{+}$cells gave significant results in patients affected by SSc-related ILD. On the contrary, no significant results were obtained when investigating $\mathrm{CD} 80^{+} \mathrm{CD} 86^{+}$ cells as initial gating strategy, thus confirming previous studies demonstrating a prevalent presence of markers characteristically linked with the alternately activated macrophage phenotype in SSc patients [31, 32].

The cellular subsets that appear to be correlated with pulmonary involvement seem to be essentially two: the first characterized by the positivity for CD204 and other M2 and M1 surface markers but negative for CD14; the second subset made up of cells positive for CD14 and for other M1 and M2 surface markers, possibly less mature than those positive for CD204. As described by 


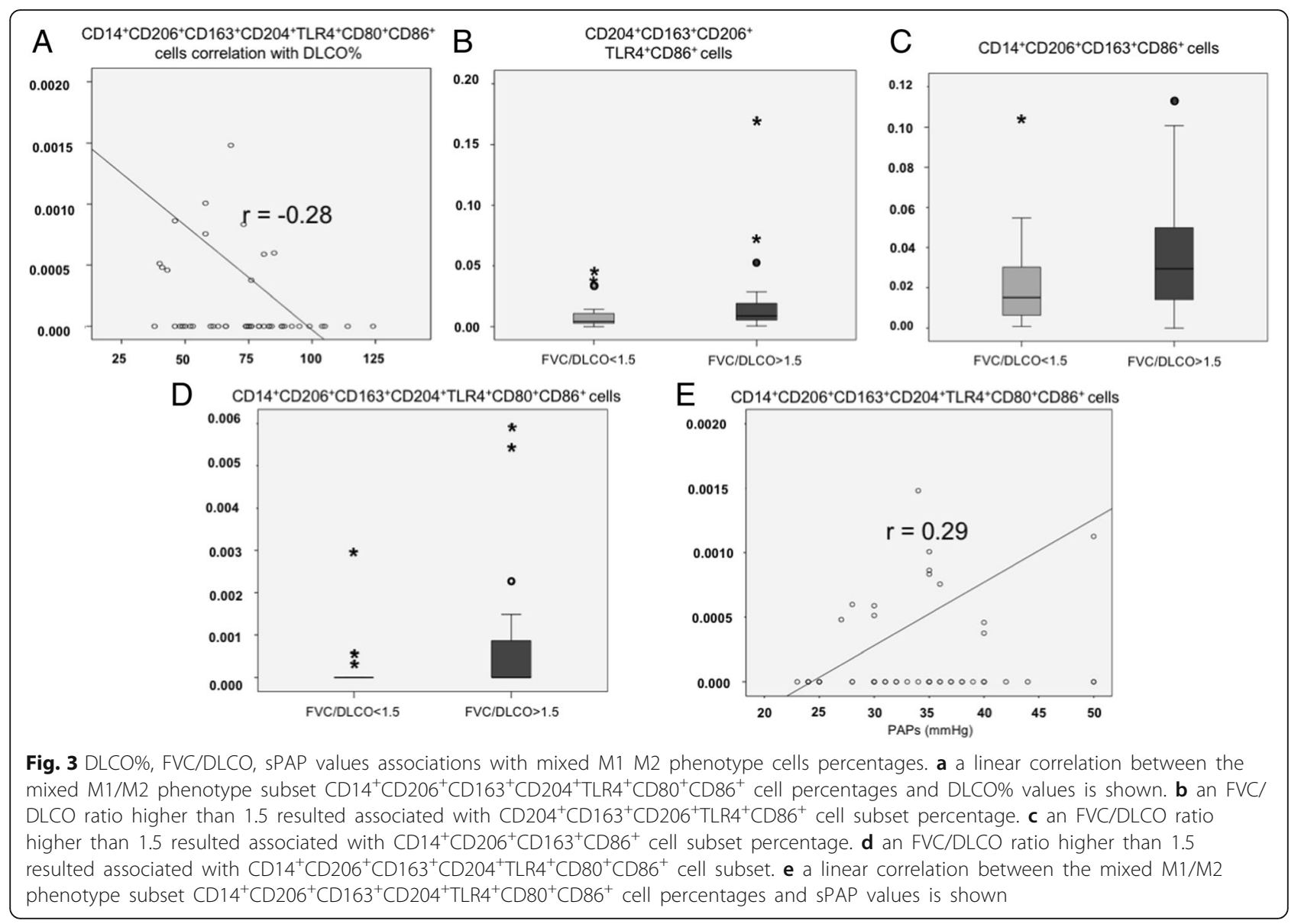

Lambert et al., although the mature circulating monocytes are classically characterized by their expression of CD14, this marker seems not to be considered the hallmark for monocyte identification, in particular in the late phase of maturation, which is accompanied by the expression of CD16 [27]. Moreover, the $\mathrm{CD} 14^{\text {low }} \mathrm{CD} 16^{+}$ monocyte subset seems to correspond to M2 monocytes/macrophages. Interestingly, in a recent study by Lescoat et al., CD16 ${ }^{+}$monocytes resulted not only associated with pulmonary fibrosis, severity of the restrictive disease and reduction of DLCO but also they were described as precursors of M2 macrophages [33]. Accordingly, even if CD16 was not evaluated in our present study, it may be possible to speculate that the mixed M1/M2 population derived from $\mathrm{CD}_{20} 4^{+}$monocytes, which is $\mathrm{CD} 14^{-}$and associated with ILD, might be positive for CD16.

The presented findings could open to the perspective of a possible role of mixed M1/M2 cells in SSc and SSc-associated ILD pathogenesis, or at least as potential biomarkers for lung involvement in SSc. In a recent study, an integrated genomic approach using a consensus clustering was performed to compare the gene expression profiles of SSc biopsies from different tissues, including skin, lung and peripheral blood mononuclear cells. The authors described the concept of the immune-fibrotic axis, that is a common pathogenic gene expression signature indicative of the fundamental role of macrophages [34].

In particular, a distinct macrophage signature associated with the alternative activation was observed in SSc-associated pulmonary fibrosis and in the skin of patients with an "inflammatory" SSc gene expression signature, suggesting that there are subtle differences in the macrophage gene expression in lung and skin [34]. Based on these observations, the authors concluded that the plasticity of the monocyte/macrophage lineage is likely to be central to the divergence of fibrotic processes in different SSc-affected tissues and is an important component of an immune-fibrotic axis driving disease pathogenesis [34].

At the same time, the statistics from the present study show the constant presence, in patients affected or not by ILD, of a great variability of cell percentages obtained from the analysis of the various circulating monocyte/ macrophages subsets, testified by large standard deviations, and reflecting considerable heterogeneity in cell size. Although this phenomenon could be attributed to 
the limited sample size, it may also be related to the cells phenotype plasticity in relation with different environmental stimuli and to their capacity to rapidly change accordingly, resulting in wide distribution of surface markers positivity at the time of the analysis [20].

Several authors have focused on how macrophage phenotype modifications could contribute to the development of lung fibrotic and neoplastic disorders [14, 16]. However, the studies often concentrated on the expression of single phenotype surface markers or soluble molecules and their possible association with diseases clinical features. It is the case, for example, of the presence of the M2 markers CD163 or soluble (s)CD163 that showed to be higher in presence of several organ involvements, like in ILD and pulmonary arterial hypertension (PAH), in SSc and other autoimmune diseases, like polymyositis and dermatomyositis [28, 29].

Serum and urinary sCD163 concentrations were very recently investigated as possible biomarkers in SSc patients compared to HSs and a study demonstrated that serum sCD163 levels were significantly higher in SSc patients compared to HSs [35]. However, sCD163 concentrations were not associated with clinical, laboratory, and instrumental characteristics of SSc patients [35].

Other authors described M2 macrophages in SSc patients and their association with several clinical parameters. Higher percentages of circulating cells positive for CD204, CD163, and CD14 were shown to correlate with skin involvement [31].

Circulating alternatively activated $\mathrm{CD}^{+} 4^{+}$macrophages, expressing high levels of CD206 were demonstrated to be associated with PAH [32].

A recent study investigated the phenotype of human alveolar macrophages (AMs) in adults living in UK and Malawi, demonstrating that the majority of AMs expressed high levels of M1 and M2 markers simultaneously. As the authors postulate, it is possible that in the healthy lung mucosa, combined M1/M2 features could confer to AMs the ability of maintaining a balance between immune tolerance and protective immunity. On the contrary, a similar circulating phenotype could exert a pathogenic role in SSc patients [21].

Moreover, it was demonstrated that monocyte-derived macrophages co-expressing CD206, CD163 and CD169 were significantly higher in SSc-ILD than in lung cancer or sarcoidosis and that a similar macrophage phenotype was obtained from the analysis of blood-monocytes derived macrophages in SSc patients [36].

However, to our knowledge, no one has so far attempted such a wide phenotype characterization of circulating monocytes/macrophages in connection with the development of SSc-related lung and right heart complications.

This study has several limits. First of all, the relatively small patient population. In fact, even if in a previous paper an effect of treatment regimens on circulating monocyte/macrophage phenotype was observed [22], in the present study a further subgroup analysis of patients with lung involvement and undergoing specific treatments compared to non-treated patients, seems not to be possible without losing too much statistical power. A follow study including a larger number of SSc patients is planned. Accidentally, most of the patients in the study population had consistently high levels of FVC (average values 104\%) and very few patients had a severe restrictive disease, therefore it was not possible to ascertain the presence of a linear correlation with $\mathrm{FVC} \%$ or with its decline.

Secondarily, the evaluation of only circulating cells is also a limitation. In fact, while interpreting the presented results, it should be kept in mind that a wide debate is taking place on the possible link between circulating and tissue resident monocyte/macrophage cells, questioning the dogma on the monocyte origin of tissue macrophages. Infiltrating macrophages, observed in the diseased tissue, seem to derive from circulating monocytes, but several studies opposed the monocyte origin for tissue resident cells [37, 38].

Moreover, emerging immunological theories attribute to organ and tissues the control of immune system activation and its forms (Th1 or Th2 like) [39]. Although the surface markers investigated in the study (primarily CD204, CD163 and CD206) are considered specific for the characterization of M2 polarized cells, they can also be expressed by dendritic cells $[40,41]$. Therefore, based on the gating strategies proposed in our study, a percentage of dendritic cells might be present in the described circulating cell subsets. Monocytes, macrophages and dendritic cells are all members of the mononuclear phagocyte system which is involved in multiple functions during immune responses and, although these cells may be distinguished based on functional and phenotypical characteristics, some cell features are often overlapping and the distinction or classification is challenging [42]. The possible implication of dendritic cells in the development of SSc was recently highlighted in a recent paper by Silvan et al. describing that these cells expressing high levels of PSGL-1 were associated with the presence of interstitial lung disease in SSc patients [43].

Finally, the very low percentage of the newly described circulating cells could make it not easy to use them as a disease biomarker. Evidently, further and larger studies and possibly the sorting of the mixed M1/M2 population will be useful for the evaluation of the importance of this phenotype in both pathogenic, diagnostic or therapeutic perspectives.

Although the identification of these circulating mixed M1/M2 cells was performed through the 
evaluation of the most investigated and specific markers related to each polarization status and in a previous study they were found significantly increased in SSc patients compared to HSs, the analysis of the marker expression through the detection of the mean fluorescence intensity (MFI), not investigated in this study, might represent a further important aspect that may contribute to understand their possible role in SSc pathogenesis.

In accordance with our results on a possible circulating "scleroderma" macrophage with M1/M2 phenotype, very recently, Moreno-Moral et al. have found in $57 \mathrm{SSc}$ patients, through RNA sequencing and genome-wide genotyping, a mixed macrophage activation signature, characterized by the downregulation of interferon gamma response, attesting for an M2 polarization, but also by the downregulation of the interleukin (IL)-6/ JAK/STAT3 signalling pathway, suggesting for a restricted M2 activity [44]. The authors observe that the circulating monocyte/macrophage phenotype could contrast with macrophage signature in tissues such as lung, in which a STAT3-dependent expression of CD163 was associated with pulmonary fibrosis [45].

Interstitial lung disease is a major cause of morbidity and mortality in systemic sclerosis (SSc). Notwithstanding many authors concentrated their attention in this direction, the pathogenic mechanisms of SSc-related ILD remain unknown, and limited therapeutic effects are obtained with the available treatments [46-48].

The knowledge of the mechanisms that initiate the pathogenesis of pulmonary damage or of an easily evaluable biomarker associated with such involvement would be crucial.

Supported by the discovery of interferon (IFN) $\alpha$, tumor necrosis factor (TNF) $\alpha$, TLRs, transforming growth factor (TGF) $\beta$, platelet derived growth factor (PDGF), genes signatures in SSc, we hypothesized that both Th1 and Th2 activation signals could derive from different damaged tissues, determining the development of circulating mixed M1/M2 cells. At the same time, more polarized responses could possibly develop at tissues level, such as in lungs [39].

\section{Conclusions}

In conclusion, it is possible to state that this is the first study showing an association of an M1/M2 monocyte/ macrophage phenotype in SSc patients to SSc-related ILD functional and radiological data.

The evaluation of the existence of circulating mixed M1/M2 monocyte/macrophage phenotype and its clinical associations in SSc patients, should be considered as the first step towards a conclusion for a possible role as a pathogenic factor or as an early biomarker for organ involvement in SSc-related ILD. Such a phenotype could be found also in other pulmonary diseases, at the circulatory or tissue level. The presented acquisitions could therefore be considered as an opening to later studies on a wide phenotype characterization of macrophages at the level of different diseased tissues including lung, kidney, heart, and skin in SSc but also in other fibrotic disorders. Furthermore, isolation and functional study of the described cells are under evaluation for the remarkable values they could have for physiopathology, diagnostic and therapeutic purposes [49].

\section{Additional files}

Additional file 1: Gating strategies for the detection of circulating M1, M2 and mixed M1/M2 cells in systemic sclerosis patients and healthy controls. (A) Representative flow cytometry scatter plot and scatter dot plot with median and interquartile range of the initial gating strategy starting from the circulating CD14 $4^{+}$cells percentage (\%) in the leucocyte population; (B) Representative flow cytometry panels with quadrant regions and scatter dot plot representation of the of circulating $\mathrm{CD}_{14}{ }^{+} \mathrm{CD} 206^{+} \mathrm{CD}_{163}{ }^{+}$cells in the $\mathrm{CD} 14^{+}$cell population; (C)

$\mathrm{CD}_{14}{ }^{+} \mathrm{CD} 206^{+} \mathrm{CD}_{163}{ }^{+} \mathrm{CD} 204^{+} \mathrm{TLR}^{+}$cells in the $\mathrm{CD} 14^{+} \mathrm{CD} 206^{+} \mathrm{CD} 163^{+}$cell subset and (D) $\mathrm{CD} 14^{+} \mathrm{CD} 206^{+} \mathrm{CD} 163^{+} \mathrm{CD} 204^{+} \mathrm{TLR}^{+} \mathrm{CD} 80^{+} \mathrm{CD} 86^{+}$cells in the $\mathrm{CD} 14^{+} \mathrm{CD} 206^{+} \mathrm{CD} 163^{+} \mathrm{TLR}^{+}$cell subset of healthy subjects (HSs) and systemic sclerosis patients (SSc pts). (E) Representative flow cytometry scatter plot and scatter dot plot with median and interquartile range of the initial gating strategy starting from the circulating $\mathrm{CD}_{20} 4^{+}$cells percentage (\%) in the leucocyte population; (F) Representative flow cytometry panels with quadrant regions and scatter dot plot representation of the of circulating $\mathrm{CD} 204^{+} \mathrm{CD} 163^{+} \mathrm{CD} 206^{+}$cells in the

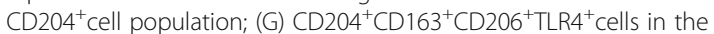

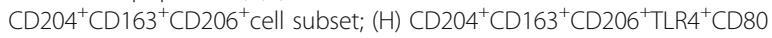
${ }^{+} \mathrm{CD} 86^{+}$cells and (I) CD14 ${ }^{+}$and $\mathrm{CD} 14^{-}$cells in the CD204 ${ }^{+} 163^{+} \mathrm{CD} 206$ ${ }^{+} \mathrm{TLR}^{+}$cell subset of HSs and SSc pts. (J) Representative flow cytometry scatter plot of the initial gating strategy starting from the circulating CD80 + CD86 + cells percentage (\%) in the leucocyte population and (L) representative flow cytometry panels with quadrant regions of the of circulating $\mathrm{CD} 80^{+} \mathrm{CD} 86^{+} \mathrm{TLR} 2^{+} \mathrm{TLR} 4^{+}$cells in the $\mathrm{CD} 80^{+} \mathrm{CD} 86^{+}$cell population of HSs and SSc pts. Statistical analysis was performed by Mann-Whitney non-parametric test and $p$-values lower than 0.05 was considered as statistically significant. (TIF $1646 \mathrm{~kb}$ )

Additional file 2: Differences in the percentage of mixed M1/M2 cells in systemic sclerosis patients with or without Ab anti Scl70 positivity and healthy subjects. Cell populations with a mixed M1/M2 phenotype, showing significantly different percentages between Scl70 antibody positive (Scl70 + Pts) and Scl70 antibody negative (Scl70-Pts) patients at Mann-Whitney were then analyzed together with those from age and gender matched healthy subjects (HSs) through Kruskal-Wallis test. HSs showed constantly lower percentages compared to Scl70 + Pts and Scl70-Pts. (TIF $514 \mathrm{~kb}$ )

Additional file 3: Differences in the percentage of $\mathrm{M} 2$ and mixed M1/ M2 cells in systemic sclerosis patients with an FVC/DLCO ratio lower or higher than 1.5. With both gating strategies, one based on CD204 positivity and one based on CD14 positivity, cell populations with an M2 or a mixed M1/M2 phenotype, showed significantly higher percentages in patients with an FVC/DLCO ratio higher then 1.5 compared to patients with an FVC/DLCO ratio lower than 1.5. (DOCX $13 \mathrm{~kb}$ )

\section{Abbreviations}

Ab anti-Scl70: anti-topoisomerase antibody; ACA: Anti-centromere antibodies; ACR: American College of Rheumatology; ANA: Anti-nuclear antibody; CT: Computed tomography;i dcSSc: Diffused cutaneous systemic sclerosis; DLCO: Diffusing capacity of the lungs for carbon monoxide; EULAR: European League Against Rheumatism; FVC: Forced vital capacity; IL: Interleukin; ILD: Interstitial lung disease; ICSSc: Limited cutaneous systemic sclerosis; MRC1: Gene codifying for c-type mannose receptor 1; NSIP: Nonspecific interstitial pneumonia; PFTs: Pulmonary function tests; pro-BNP: Pro- 
hormone of brain natriuretic peptide; PTs: Patients; RP: Raynaud's phenomenon; SD: Standard deviation; SPAP: Systolic pulmonary artery pressure; SSc: Systemic sclerosis; Th: T helper; TLR: Toll-like receptors

\section{Acknowledgements}

We acknowledge Sara de Gregorio supervising the graphic representation of the data.

\section{Funding}

This research did not receive any specific grant from funding agencies in the public, commercial, or not-for-profit sectors.

\section{Availability of data and materials}

The datasets used and/or analysed during the current study are available from the corresponding author on reasonable request.

\section{Authors' contributions}

$A C T^{*}$ designed the study, carried out the statistical analysis and wrote the manuscript, SS* designed the study, analysed the data and wrote the manuscript; $\mathrm{PC}$ performed the Flow Cytometry analysis, VT and BR enrolled the systemic sclerosis patients and collected the demographic and clinical parameters of the systemic sclerosis patients; RB and PM managed and prepared the samples for the Flow Cytometry analysis; AS, SP and CP supervised the enrolment of the systemic sclerosis patients and healthy subjects and the clinical data collection; VS revised the manuscript; MC supervised the study, wrote and revised the final manuscript. All authors read and approved the final manuscript.

\section{Ethics approval and consent to participate}

The study was approved by the Ethics Commitee of Polyclinic San Martino Hospital, Genoa, Italy (protocol number: 273-reg-2015). All patients gave a written informed consent before enrollment.

\section{Consent for publication}

Not applicable.

\section{Competing interests}

The authors declare that they have no competing interests.

\section{Publisher's Note}

Springer Nature remains neutral with regard to jurisdictional claims in published maps and institutional affiliations.

\section{Author details}

${ }^{1}$ Research Laboratory and Academic Division of Clinical Rheumatology, Department of Internal Medicine, University of Genova, Polyclinic San Martino Hospital, Genoa, Italy. ${ }^{2}$ Clinical Immunology, Department of Internal Medicine, University of Genova, Genoa, Italy. ${ }^{3}$ Department of Rheumatology, Ghent University Hospital, Ghent, Belgium. ${ }^{4}$ Department of Internal Medicine, Ghent University, Ghent, Belgium.

Received: 4 May 2018 Accepted: 12 September 2018 Published online: 24 September 2018

\section{References}

1. Smith V, Riccieri V, Pizzorni C, Decuman S, Deschepper E, Bonroy C, et al Nailfold capillaroscopy for prediction of novel future severe organ involvement in systemic sclerosis. J Rheumatol. 2013:40:2023-8.

2. Elhai M, Meune C, Boubaya M, Avouac J, Hachulla E, Balbir-Gurman A, et al. Mapping and predicting mortality from systemic sclerosis. Ann Rheum Dis. 2017;76:1897-905.

3. Taichman DB, Shin J, Hud L, Archer-Chicko C, Kaplan S, Sager JS, et al. Health-related quality of life in patients with pulmonary arterial hypertension. Respir Res. 2005;6:92.

4. Hachulla E, Carpentier P, Gressin V, Diot E, Allanore Y, Sibilia J, et al. Risk factors for death and the 3-year survival of patients with systemic sclerosis: the French ItinerAIR-Sclerodermie study. Rheumatology (Oxford). 2009;48:304-8.

5. Zhao J, Wang Q, Liu Y, Tian Z, Guo X, Wang H, et al. Clinical characteristics and survival of pulmonary arterial hypertension associated with three major connective tissue diseases: a cohort study in China. Int J Cardiol. 2017;236:432-7.
6. Goh NS, Desai SR, Veeraraghavan S, Hansell DM, Copley SJ, Maher TM, et al. Interstitial lung disease in systemic sclerosis: a simple staging system. Am J Respir Crit Care Med. 2008;177:1248-54.

7. Luo Y, Wang Y, Wang Q, Xiao R, Lu Q. Systemic sclerosis: genetics and epigenetics. J Autoimmun. 2013;41:161-7.

8. Gourh P, Agarwal SK, Martin E, Divecha D, Rueda B, Bunting H, et al. Association of the C8orf13-BLK region with systemic sclerosis in northAmerican and European populations. J Autoimmun. 2010;34:155-62.

9. Christmann RB, Sampaio-Barros P, Stifano G, Borges CL, de Carvalho CR, Kairalla $R$, et al. Association of Interferon- and transforming growth factor beta-regulated genes and macrophage activation with systemic sclerosisrelated progressive lung fibrosis. Arthritis Rheumatol. 2014;66:714-25.

10. Hsu E, Shi H, Jordan RM, Lyons-Weiler J, Pilewski JM, Feghali-Bostwick CA. Lung tissues in patients with systemic sclerosis have gene expression patterns unique to pulmonary fibrosis and pulmonary hypertension. Arthritis Rheum. 2011;63:783-94.

11. Enomoto Y, Suzuki Y, Hozumi H, Mori K, Kono M, Karayama M, et al. Clinical significance of soluble CD163 in polymyositis-related or dermatomyositisrelated interstitial lung disease. Arthritis Res Ther. 2017;19:9.

12. Jiang Z, Zhu L. Update on the role of alternatively activated macrophages in asthma. J Asthma Allergy. 2016;9:101-7.

13. Bazzan E, Turato G, Tinè M, Radu CM, Balestro E, Rigobello C, et al. Dual polarization of human alveolar macrophages progressively increases with smoking and COPD severity. Respir Res. 2017;18:40.

14. Groves AM, Johnston CJ, Misra RS, Williams JP, Finkelstein JN. Effects of IL-4 on pulmonary fibrosis and the accumulation and phenotype of macrophage subpopulations following thoracic irradiation. Int J Radiat Biol. 2016;92:754-65.

15. Deckman JM, Kurkjian CJ, McGillis JP, Cory TJ, Birket SE, Schutzman LM, et al. Pneumocystis infection alters the activation state of pulmonary macrophages. Immunobiology. 2017;222:188-97.

16. Huang F, Chen Z, Chen H, Lu W, Xie S, Meng QH, et al. Cypermethrin promotes lung Cancer metastasis via modulation of macrophage polarization by targeting MicroRNA-155/Bcl6. Toxicol Sci. 2018. https://doi. org/10.1093/toxsci/kfy039 [Epub ahead of print].

17. Mantovani A, Biswas SK, Galdiero MR, Sica A, Locati M. Macrophage plasticity and polarization in tissue repair and remodelling. J Pathol. 2013;229:176-85.

18. Stifano G, Christmann RB. Macrophage involvement in systemic sclerosis: do we need more evidence? Curr Rheumatol Rep. 2016;18:2

19. Gundra UM, Girgis NM, Ruckerl D, Jenkins S, Ward LN, Kurtz ZD, et al. Alternatively activated macrophages derived from monocytes and tissue macrophages are phenotypically and functionally distinct. Blood. 2014;123:e110-22.

20. Mosser DM, Edwards JP. Exploring the full spectrum of macrophage activation. Nat Rev Immunol. 2008:8:958-69.

21. Mitsi E, Kamng'ona R, Rylance J, Solórzano C, Jesus Reiné J, Mwandumba $\mathrm{HC}$, et al. Human alveolar macrophages predominately express combined classical M1 and M2 surface markers in steady state. Respir Res. 2018;19:66.

22. Soldano S, Trombetta AC, Contini P, Tomatis V, Ruaro B, Brizzolara R, et al. Increase in circulating cells coexpressing M1 and M2 macrophage surface markers in patients with systemic sclerosis. Ann Rheum Dis Epub ahead of print. Ann Rheum Dis 2018;0:1-3. doi:https:/doi.org/10.1136/annrheumdis-2018-213648.

23. van den Hoogen F, Khanna D, Fransen J, Johnson SR, Baron M, Tyndall A, et al. 2013 classification criteria for systemic sclerosis: an American college of rheumatology/European league against rheumatism collaborative initiative. Ann Rheum Dis. 2013;65:2737-47.

24. Hudson $M$, Fritzler MJ. Diagnostic criteria of systemic sclerosis. J Autoimmun. 2014;48-49:38-41.

25. Kim EA, Johkoh T, Lee KS, Ichikado K, Koh EM, Kim TS, et al. Interstitial pneumonia in progressive systemic sclerosis: serial high-resolution CT findings with functional correlation. J Comput Assist Tomogr. 2001;25:757-63.

26. Sivova N, Launay D, Wémeau-Stervinou L, De Groote P, Remy-Jardin M, Denis $\mathrm{G}$, et al. Relevance of partitioning DLCO to detect pulmonary hypertension in systemic sclerosis. PLoS One. 2013;8:e78001.

27. Lambert C, Preijers FWMB, Yanikkaya Demirel G, Sack U. Monocytes and macrophages in flow: an ESCCA initiative on advanced analysis of monocyte lineage using flow cytometry. Cytometry Part B. 2017;92:180-8.

28. Arroz M, Came N, Lin P, Chen W, Yuan C, Lagoo A, et al. Consensus guidelines on plasma cell myeloma minimal residual disease analysis and reporting. Cytometry Part B. 2016;90:31-9.

29. Briggs DC, Vaughan RW, Welsh Kl, Myers A, DuBois RM, Black CM. Immunogenetic prediction of pulmonary fibrosis in systemic sclerosis. Lancet. 1991;338:661-2. 
30. Hu PQ, Oppenheim JJ, Medsger TA Jr, Wright TM. T cell lines from systemic sclerosis patients and healthy controls recognize multiple epitopes on DNA topoisomerase. J Autoimmun. 2006;26:258-67.

31. Higashi-Kuwata N, Jinnin M, Makino T, Fukushima S, Inoue Y, Muchemwa FC, et al. Characterization of monocyte/macrophage subsets in the skin and peripheral blood derived from patients with systemic sclerosis. Arthritis Res Ther. 2010;12:R128.

32. Christmann RB, Hayes E, Pendergrass S, Padilla C, Farina G, Affandi AJ, et al. Interferon and alternative activation of monocyte/macrophages in systemic sclerosis-associated pulmonary arterial hypertension. Arthritis Rheum. 2011; 63:1718-28.

33. Lescoat A, Lecureur V, Roussel M, Sunnaram BL, Ballerie A, Coiffier G, et al. CD16-positive circulating monocytes and fibrotic manifestations of systemic sclerosis. Clin Rheumatol. 2017;36:1649-54.

34. Taroni JN, Greene CS, Martyanov V, Wood TA, Christmann RB, Farber HW, et al. A novel multi-network approach reveals tissue-specific cellular modulators of fibrosis in systemic sclerosis. Genome Med. 2017:9:27.

35. Frantz C, Pezet S, Avouac J, Allanore Y. Soluble CD163 as a potential biomarker in systemic sclerosis. Dis Markers. 2018;2018:8509583.

36. Lescoat A, Ballerie A, Augagneur Y, Morzadec C, Vernhet L, Fardel O, et al. Distinct properties of human M-CSF and GM-CSF monocyte-derived macrophages to simulate pathological lung conditions in vitro: application to systemic and inflammatory disorders with pulmonary involvement. Int J Mol Sci 2018:19. pii: E894. doi: https://doi.org/10.3390/ijms19030894.

37. Qian BZ, Li J, Zhang H, Kitamura T, Zhang J, Campion LR, et al. CCL2 recruits inflammatory monocytes to facilitate breast-tumour metastasis. Nature. 2011;475:222-5.

38. Chawla A, Nguyen KD, Goh YP. Macrophage-mediated inflammation in metabolic disease. Nat Rev Immunol. 2011;11:738-49.

39. Matzinger P, Kamala T. Tissue-based class control: the other side of tolerance. Nat Rev Immunol. 2011;11:221-30.

40. Yu X, Wang XY. Antagonizing the innate pattern recognition receptor CD204 to improve dendritic cell-targeted cancer immunotherapy. Oncoimmunology. 2012;1:770-2.

41. Collin M, McGovern N, Haniffa M. Human dendritic cell subsets. Immunology. 2013;140:22-30.

42. Guilliams M, Ginhoux F, Jakubzick C, Naik S, Onai N, Schraml B, et al. Dendritic cells, monocytes and macrophages: a unified nomenclature based on ontogeny. Nat Rev Immunol. 2014;14:571-8.

43. Silván J, González-Tajuelo R, Vicente-Rabaneda E, et al. Deregulated PSGL-1 expression in B cells and dendritic cells may be implicated in human systemic sclerosis development. J Invest Dermatol. 2018;22.

44. Moreno-Moral A, Bagnati M, Koturan S, Ko JH, Fonseca C, Harmston N, et al. Changes in macrophage transcriptome associate with systemic sclerosis and mediate GSDMA contribution to disease risk. Ann Rheum Dis. 2018;77:596-601.

45. Lescoat A, Jégo P, Lecureur V. M-CSF and GM-CSF monocyte-derived macrophages in systemic sclerosis: the two sides of the same coin? Ann Rheum Dis. 2018. https://doi.org/10.1136/annrheumdis-2018-213112 [Epub ahead of print].

46. Johnson ME, Mahoney JM, Taroni J, Sargent JL, Marmarelis E, Wu MR, et al. Experimentally-derived fibroblast gene signatures identify molecular pathways associated with distinct subsets of systemic sclerosis patients in three independent cohorts. PLoS One. 2015;10:e0114017.

47. du Bois RM. Mechanisms of scleroderma-induced lung disease. Proc Am Thorac Soc. 2007:4:434-8.

48. Tan A, Denton CP, Mikhailidis DP, Seifalian AM. Recent advances in the diagnosis and treatment of interstitial lung disease in systemic sclerosis (scleroderma): a review. Clin Exp Rheumatol. 2011;29:566-74.

49. Burmester GR, Bijlsma JWJ, Cutolo M, Mclnnes IB. Managing rheumatic and musculoskeletal diseases - past, present and future. Nat Rev Rheumatol. 2017:13:443-8

Ready to submit your research? Choose BMC and benefit from:

- fast, convenient online submission

- thorough peer review by experienced researchers in your field

- rapid publication on acceptance

- support for research data, including large and complex data types

- gold Open Access which fosters wider collaboration and increased citations

- maximum visibility for your research: over $100 \mathrm{M}$ website views per year

At BMC, research is always in progress.

Learn more biomedcentral.com/submissions 